Original Article

\title{
DDA LOADED PCL NANOPARTICLES ENHANCES THE ORAL BIOAVAILABILITY OF DDA IN DIABETES INDUCED EXPERIMENTAL RATS
}

\author{
NAGALAKSHMI K. a , SUJATHA S. a $^{*}$, ALWIN D. \\ aDepartment of Biotechnology, School of Bioengineering, SRM University, Kattankulathur 603203, bCentral Animal House, SRM Medical \\ College Hospital and Research Centre, SRM University, Kattankulathur 603203 \\ Email: sujatha.sa@ktr.srmuniv.ac.in \\ Received: 30 Dec 2016 Revised and Accepted: 02 Mar 2017
}

\begin{abstract}
Objective: The present study was designed to evaluate the bioavailability of nano encapsulated DDA (nano-DDA) in experimental diabetic rats.

Methods: Polycaprolactone was used as a polymer to encapsulate 14-deoxy-11, 12-didehydroandrographolide (DDA) using solvent evaporation technique in order to improvise the bioavailability of the drug. Male albino wistar rats were induced with single intraperitoneal injection of nicotinamide $(110 \mathrm{mg} / \mathrm{kg})$ followed by streptozotocin $(45 \mathrm{mg} / \mathrm{kg})$ to induce experimental diabetes. Free DDA and nano-DDA were orally administered to the experimental diabetic rats for $45 \mathrm{~d}$ and blood glucose level was monitored periodically. After one week washout period, free DDA and nano-DDA were orally administered to the rats and blood samples were collected at predetermined intervals. Plasma concentration of DDA was determined by highperformance liquid chromatography (HPLC). Pharmacokinetic analysis was carried out to determine the oral bioavailability.
\end{abstract}

Results: $50 \mathrm{mg}$ of nanoparticle-containing $9.4 \mathrm{mg}$ of DDA exhibited a significant decrease in blood glucose level (105.6 $\pm 2.99 \mathrm{mg} / \mathrm{dL}$ ), on par with the free drug administered ( $50 \mathrm{mg} / \mathrm{kg}$ ). The nano-DDA accomplished a significant increase in $\mathrm{C}_{\max }(961.7 \pm 8.78 \mathrm{ng}$ ) and area under the curve (AUC) $(2631 \pm 6.98 \mathrm{~h} \mathrm{X} \mathrm{ng} / \mathrm{ml})$ than free DDA. A significant increase in the oral bioavailability was witnessed for nano-DDA (absolute bioavailability\% $=$ $34.94 \pm 0.231 \%)$, which was 10.8 times higher than the free DDA $(3.234 \pm 0.062 \%)$ and substantiated a slow and sustained drug release from the polymer matrix.

Conclusion: Our results substantiated that nanoencapsulation of DDA, enhanced the oral bioavailability of DDA than the free drug in vivo. NanoDDA can thus serve as a bioactive molecule in the quest for new antidiabetic nano drug discovery.

Keywords: DDA, Nano-DDA, Nanoencapsulation, Oral bioavailability, Antidiabetic, Drug release, HPLC

(c) 2017 The Authors. Published by Innovare Academic Sciences Pvt Ltd. This is an open access article under the CC BY license (http://creativecommons.org/licenses/by/4.0/] DOI: http://dx.doi.org/10.22159/ijpps.2017v9i4.16860

\section{INTRODUCTION}

14-deoxy-11, 12-didehydroandrographolide (DDA) is a diterpenoid, of the annual herbaceous plant Andrographis paniculata (Family: Acanthaceae) [1]. It is one of the herbal medicine which has been used for centuries in India and other asian countries to treat several diseases such as gastrointestinal tract, upper respiratory tract infections and herpes [2]. The leaves and aerial parts of the plant have been used to cure various ailments. The major constituent that imparts the bitter taste to this herb is andrographolide.

Other potential diterpenoid constituents include neo andrographolide, 14-deoxy-11, 12-didehydro andrographolide and 14deoxy andrographolide [3]. The extracts of $A$. paniculata and its isolated compounds were also reported to have plethora of pharmacological activities. 14-deoxy-11, 12-didehydro andrographolide (DDA) exhibited a pronounced antidiabetic activity in our previous studies both in vitro and in vivo models (unpublished data). In addition to that, DDA demonstrated potent cardiovascular effects [4], vasorelaxing effect [5] and anti-diabetic nephropathy effect [6].

Many studies reported the pharmacokinetic studies of $A$. paniculata in rats as well as in humans $[7,8]$. Few studies reported the pharmacokinetic parameters of the andrographolide and not much of other diterpenoids of the plant. The less availability of pharmacokinetic data of the bioactive compound might result in the delay of further drug development and clinical uses.

In drug discovery, poor water solubility is the major issue for various new chemical entities [9]. Though extensive research and development have been done, less solubility of bioactive principles in aqueous solution continued as a setback for their bioavailability and clinical application. Exploring a safe and effective method for solubilizing sparingly water soluble drugs remained an active part of pharmaceutical research [3]. Many approaches have been investigated to improve the oral bioavailability of the drug by augmenting drug solubility or increasing the surface area available for dissolution [4]. So far, attempts have been made to encapsulate these compounds into the nanoparticulate system so as to achieve the targeting ability and delivery of drug at the target site, thereby increasing its solubility and bioavailability in the form of liposomes, polymeric and lipo nanoparticles, biodegradable microspheres, cyclodextrin and hydrogels.

Oral administration of drugs plays a crucial role in the development of pharmaceutical research due to its extensive clinical applications. With the aim to improve the oral bioavailability, several strategies have been proposed to reduce dosing frequency and/or gastrointestinal side-effects of many drugs.

Though DDA has shown antidiabetic activity in vitro, due to its poor water solubility could not show a pronounced antihyperglycemic activity in vivo (data not shown). Therefore our study aimed at the nanoencapsulation of DDA using PCL (nano-DDA) so as to improvise targeted drug delivery.

The present study aims at evaluating the bioavailability and pharmacokinetic changes of DDA and nano-DDA after their oral administration in experimental rats.

\section{MATERIALS AND METHODS}

\section{Materials}

14-deoxy, 11, 12 didehydro andrographolide was purchased from Apeksha Research Centre private limited, Indore, Madhya Pradesh, India. All other chemicals and reagents used unless otherwise stated were of analytical grade. 


\section{Nanoparticles}

Formulation and characterization of DDA loaded PCL nanoparticles

The nanoparticles were synthesized using solvent evaporation technique and characterized using scanning electron microscopy, HPLC, Fourier transform infrared spectroscopy (FTIR), in vitro drug release and cellular uptake studies [10].

\section{Animal experiments and drug administration}

Healthy male albino Wistar rats, weighing 150-200 g of 7-8 w were procured from Tamilnadu Veterinary University, Madhavaram, Chennai. The experimental protocol was approved by the Institutional animal ethical committee (Approval number: IAEC 1543,2011 ) and was maintained as per the guidelines of the National institute of nutrition (NIN), Indian council of medical research (ICMR), Hyderabad, India. The animals were fed with normal laboratory pellet diet and water ad libitum. During the experimental period, animals were housed in polypropylene cages lined with husk in standard environmental conditions (temperature $25 \pm 2^{\circ} \mathrm{C}$, relative humidity $55 \pm 10 \%$ and $12: 12$ light: dark cycle). Ethical guidelines were strictly followed during the study.

\section{Experimental induction of diabetes}

Diabetes was induced in overnight fasted rats by single intraperitoneal injection (i. p.) of freshly dissolved streptozotocin ( $45 \mathrm{mg} / \mathrm{kg}$ ) prepared in cold citrate buffer $\mathrm{pH} \mathrm{4.5,} 15 \mathrm{~min}$ after the i. p. injection of nicotinamide $(110 \mathrm{mg} / \mathrm{kg})$ prepared in normal physiological saline [11]. Hyperglycemia was confirmed by the elevated glucose levels in serum, determined at $3^{\text {rd }} \mathrm{d}$ and $7^{\text {th }} \mathrm{d}$ after induction [12]. The rats with sustained blood glucose levels ranging from 200 to $300 \mathrm{mg}$ glucose/dL were considered as diabetic and used for the study.

\section{Drug administration}

Before initiating the experiment, animals were given one week washout period to remove traces of the drug. Free and nano-DDA dosage were chosen based on the previous pilot study carried out in our laboratory. Accordingly, free DDA (50 mg/kg) was administered to the experimental rats. For the administration of nano-DDA, the actual content of DDA present in the Nano encapsulation matrix was determined using HPLC analysis. Accordingly, $50 \mathrm{mg} / \mathrm{kg}$ of nanoDDA containing $9.4 \mathrm{mg}$ of DDA (w/w) was administered to the experimental rats. The blood samples were collected from inner canthus of the rats at pre-determined time intervals.

The drug for oral administration was prepared by dissolving free DDA $(50 \mathrm{mg} / \mathrm{kg})$ in carboxymethyl cellulose $(0.5 \%)(1 \mathrm{ml})$. Nano-DDA suspensions were prepared by dissolving the required nanoencapsulated dose $(9.4 \mathrm{mg} / \mathrm{kg})$ in carboxymethyl cellulose $(0.5 \%)(1 \mathrm{ml})$.

Experimental animals were grouped as follows,

Group I-Untreated control (vehicle)

Group II-Diabetic animals treated with free DDA (50 mg/kg)

Group III-Diabetic animals treated with nano-DDA (50 mg (9.4 mg $\mathrm{DDA}) / \mathrm{kg}$ )

Group IV-Diabetic animals treated with Metformin (500 mg/kg)

\section{HPLC analysis}

Plasma was separated by the centrifugation of heparinized blood at $3500 \mathrm{rpm}$ for $10 \mathrm{~min}$. Sample preparation was performed according to Wan et al. with slight modifications [13]. To each $400 \mu \mathrm{l}$ of plasma, $1 \mathrm{ml}$ of methanol was added and vortexed for $2 \mathrm{~min}$. The tube was centrifuged at 10,000 rpm for $15 \mathrm{~min}$ and the organic layer was separated. The organic solvent was evaporated and the residue was reconstituted with $1 \mathrm{ml}$ of methanol and subjected to HPLC analysis.

\section{Pharmacokinetic parameters}

Pharmacokinetic analysis was carried out by calculating the AUC of the plasma concentration $\left(C_{p}\right)$ as a function of time $(t)$ [14]. The maximum plasma concentration $\left(\mathrm{C}_{\max }\right)$ and the time needed to reach the maximum plasma concentration $\left(\mathrm{T}_{\max }\right)$ were determined through observation of the experimental data. The elimination rate constant $\left(\mathrm{K}_{\mathrm{el}}\right)$ was obtained from the terminal slope using regression analysis and the half-life $\left(\mathrm{t}_{1 / 2}\right)$ of the drug was calculated by $0.693 / \mathrm{K}_{\mathrm{el}}$. The absolute bioavailability of free and nano-DDA were calculated using the following equation:

$$
A B \%=\frac{A U C_{\text {oral }}}{A U C_{I V}} \times \frac{I V \text { dose }}{\text { oral dose }} \times 100
$$

Where $\mathrm{AB}=$ Absolute bioavailability

AUC $=$ Area under curve

IV= intravenous

\section{Statistical analysis}

Results were expressed as mean \pm SEM. The data was analysed using Graphpad Prism 5.03 statistical software (Graphpad software Inc., La Jolla, CA). Differences among the pharmacokinetic parameters were tested for significance by one-way analysis of variance (ANOVA), followed by Tukey's post hoc test. The criterion for statistical significance was $\mathrm{P}<0.05$.

\section{RESULTS AND DISCUSSION}

The diterpene of Andrographis paniculata, DDA has a molecular formula of $\mathrm{C}_{20} \mathrm{H}_{28} \mathrm{O}_{4}$ and the molecular weight of 332.43 whose molecular structure is depicted (fig. 1). In the present study, pharmacokinetic parameters of both free and nano-DDA were comparatively analysed. The use of nanoparticles to increase the bioavailability of sparingly soluble bioactives might pave a path to research towards Nano encapsulation of bioactive compounds. Nano encapsulation of DDA was done using polycaprolactone (PCL) as a biodegradable polymer $[16,17]$. PCL has been approved by Food and drug administration (FDA) and considered as one of the bestsuited polymers for entrapping the bioactives due to its availability, biodegradability, non-toxicity and biocompatibility. The degradation of PCL is comparatively much slower than other known biodegradable polymers, which made it opt for engineering slow and sustained drug delivery devices [18]. Nano encapsulation of DDA was carried out using PCL as a polymer and further characterized using SEM, HPLC, FTIR, in vitro drug release and cellular uptake studies in our earlier studies [10].

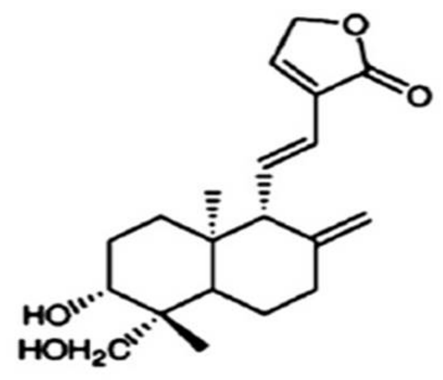

Fig. 1: Molecular structure of DDA with a molecular formula $\mathrm{C}_{20} \mathrm{H}_{28} \mathrm{O}_{4}$ and molecular weight 332.43

The amount of drug present in the various formulations of nanoDDA was displayed (table 1). Drug content expresses the amount of bioactive compound loaded in the polymer matrix. As displayed in table 1, A1 formulation showed the highest drug content loaded into the polymer matrix $(5 \mathrm{mg}$ of nano-DDA contains $0.94 \mathrm{mg}$ of DDA). Among all the formulations, A3 formulation exhibited the highest nanoparticle yield of up to $92.16 \pm 0.82 \%$, still this ratio was not preferred for further studies due to low drug content $(0.59 \pm 0.07 \mathrm{mg} / 5 \mathrm{mg})$ than the other formulations. The other two formulations A2 and A4 displayed $0.77 \pm 0.027 \mathrm{mg}$ and $0.66 \pm 0.025 \mathrm{mg}$ of drug content per 
$5 \mathrm{mg}$ respectively which was comparatively lesser than the A1 formulation. A1 formulation was prioritized due to the high drug loading content $(0.94 \pm 0.015 \mathrm{mg})$ and low drug wastage $(8.16 \pm 0.26 \%)$. The entrapment of drug into the polymer matrix depends on the drug-polymer combination and the method used for the preparation. Several factors influence the drug content including the surfactant used, ratio of polymer to drug and ratio of organic solvent to aqueous phase [19].

Table 1: Nanoparticle yield percentage, drug content, drug encapsulation efficiency and drug wastage for various formulations of drug and polymer

\begin{tabular}{llll}
\hline Formulation & $\begin{array}{l}\text { Nanoparticle } \\
\text { yield (\%) }\end{array}$ & $\begin{array}{l}\text { Drug content present in 5 mg of } \\
\text { nanoparticle (w/w) }\end{array}$ & $\begin{array}{l}\text { Drug encapsulation } \\
\text { efficiency (\%) (w/w) }\end{array}$ \\
\hline 0.5\% Polyvinyl alcohol(PVA) (A1) & $79.33 \pm 0.56$ & $0.94 \pm 0.02$ & $91.98 \pm 0.13$ \\
1\% Tween 20 (1:4) (A2) & $89.14 \pm 0.73$ & $0.77 \pm 0.03$ & $75.87 \pm 0.24$ \\
1\%Tween 20 (1:5) (A3) & $92.16 \pm 0.82$ & $0.59 \pm 0.07$ & $52.82 \pm 0.21$ \\
Double emulsion (1\% Tween & $75.16 \pm 0.59$ & $0.66 \pm 0.03$ & $63.94 \pm 0.23$ \\
20+0.5\% PVA) (A4) & & & $47.42 \pm 0.26$ \\
\hline
\end{tabular}

The data are expressed in mean $\pm \operatorname{SEM},(n=3)$

The antidiabetic effect of free and nano-DDA in diabetes-induced experimental rats was tabulated (table 2). In the study, the experimental animals were administered with $50 \mathrm{mg} / \mathrm{kg}$ of free DDA and $50 \mathrm{mg}$ ( $9.4 \mathrm{mg}$ of DDA)/kg of nano-DDA for $45 \mathrm{~d}$. In spite of the fact that nano-DDA dosage administered in the study was about 5.31 times lesser than free DDA, nano-DDA was able to exert the same effect on par with the free DDA. This result could be substantiated with the slow and sustained delivery of DDA from PCL polymer matrix, which enhanced the bioavailability of the drug thereby exhibiting the pronounced antidiabetic effect in the experimental rats.

Table 2: Effect of free and nano-DDA on blood glucose level in experimental diabetic rats

\begin{tabular}{|c|c|c|}
\hline Group & Blood glucose level at $1^{\text {st }}$ day of treatment $(\mathrm{mg} / \mathrm{dl})$ & Blood glucose level after treatment for $45 \mathrm{~d}(\mathrm{mg} / \mathrm{dL})$ \\
\hline Untreated control & $89 \pm 1.09$ & $95.4 \pm 2.4$ \\
\hline Free DDA & $395 \pm 2.96$ & $115.4 \pm 1.32 *$ \\
\hline Nano-DDA & $408 \pm 1.78$ & $105.6 \pm 2.99 *$ \\
\hline Metformin & $406 \pm 1.67$ & $107.6 \pm 2.69 *$ \\
\hline
\end{tabular}

The data are expressed in mean \pm SEM; $n=5$ in each group, * represents $\mathrm{P}<0.05$ compared with respective groups at the initial day of treatment

Bioavailability (F) represents "The relative amount of an administered dose that reaches the general circulation and the rate at which this occurs". The amount of bioactive compounds that enter into systemic circulation over time after administration can be measured by extrapolating the pharmacokinetic parameters, which generates the concentration curve of the drug in plasma at various time intervals. From the curve, AUC can be obtained and the bioavailability of various formulations can be analysed.

The plasma concentration vs. time profiles of free and nano-DDA were displayed after the oral administration of free and nano-DDA to the rats (fig. 2). The pharmacokinetic parameters of free and nano-DDA was summarized (table 3). Nano-DDA exhibited a significant $(\mathrm{P}<0.05)$ increase in the peak concentrations $\left(\mathrm{C}_{\max }\right)$ and area under the plasma concentration-time curve (AUC) as compared with the free drug. A difference in the terminal half-life $\left(t_{1 / 2}\right)$ and the time taken to reach the peak concentration $\left(\mathrm{T}_{\max }\right)$ were observed. Moreover, the absolute bioavailability ( $\mathrm{AB} \%$ ) of nano-DDA was found to be significantly higher $(34.98 \pm 0.062 \%)$ than the free DDA $(3.14 \pm 0.231 \%)(\mathrm{P}<0.05)$.

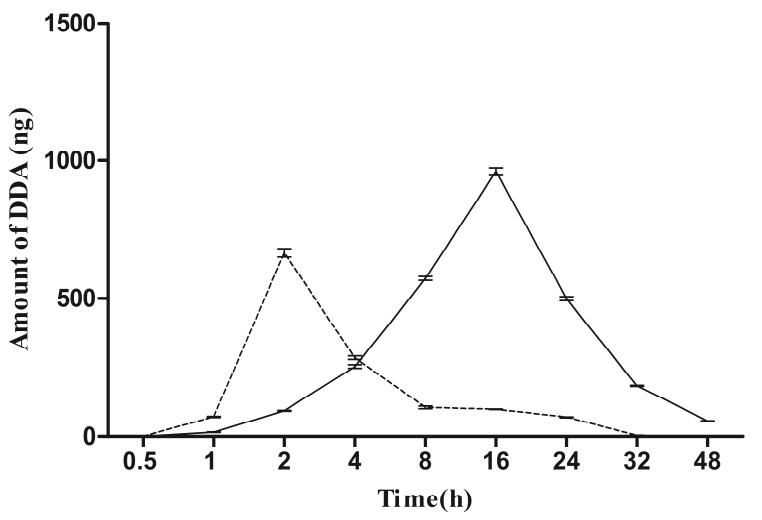

Fig. 2: Mean plasma concentration vs. time profiles of DDA. (---) free DDA (50 mg/kg), (---) nano-DDA $(50 \mathrm{mg}(9.4 \mathrm{mg}) / \mathrm{kg})$ to experimental diabetic rats. Data represented as mean $\pm S E M, n=5$

Table 3: Pharmacokinetic parameters of DDA and nano-DDA in experimental rats1

\begin{tabular}{lll}
\hline Pharmacokinetic parameters & Free DDA & nano-DDA \\
\hline Dose administered (mg/kg) & 50 & $50(9.4 \mathrm{mg}$ of DDA) \\
$\mathrm{C}_{\max }(\mathrm{ng})$ & $665.5 \pm 7.39$ & $961.7 \pm 8.78^{*}$ \\
$\mathrm{~T}_{\max }(\mathrm{h})$ & 2 & $16^{*}$ \\
$\mathrm{AUC} \mathrm{C}_{0-48 \mathrm{~h} \mathrm{ng} / \mathrm{ml}}$ & $1293 \pm 7.51$ & $2631 \pm 6.98^{*}$ \\
$\mathrm{k}(\mathrm{elimination}$ rate constant)/h & $0.103 \pm 0.04$ & $0.053 \pm 0.01^{*}$ \\
$\mathrm{t}_{1 / 2}$ (elimination half-life) $(\mathrm{h})$ & $6.729 \pm .038$ & $13.063 \pm 0.36^{*}$ \\
Absolute bioavailability (AB \%) & $3.234 \pm 0.06$ & $34.94 \pm 0.231^{*}$ \\
\hline
\end{tabular}

The data are expressed in mean \pm SEM; $n=5$ in each group, *represents $\mathrm{P}<0.05$ compared with free DDA 
The results emphasized that the oral administration of free DDA resulted in a sharp $C_{\max }(665.5 \pm 7.387 \mathrm{ng})$, early $\mathrm{T}_{\max }(2 \mathrm{~h})$ and the plasma concentration of the drug decreased rapidly, indicating a rapid metabolism of DDA. This could be attributed to the higher elimination rate $\mathrm{k}_{\mathrm{el}}(0.103 \pm 0.04 / \mathrm{h})$ and shorter $\mathrm{t}_{1 / 2}(6.729 \pm 0.038 \mathrm{~h})$. In contrast, nano-DDA displayed sustained absorption of DDA by achieving a prolonged $\mathrm{T}_{\max }$ value as compared with the free drug. The $\mathrm{C}_{\max }$ of the free DDA $(665.5 \pm 7.387 \mathrm{ng})$ and nano-DDA $(961.7 \pm 8.78 \mathrm{ng})$ also indicated that the $\mathrm{C}_{\max }$ of free DDA differs 1.44 times lesser than nano-DDA. A relatively slow increase and sustained plasma concentration of nano-DDA for a longer time were observed with significantly delayed $\mathrm{T}_{\max }(16 \mathrm{~h})$ and prolonged $\mathrm{K}_{\mathrm{el}}$ $(0.053 \pm 0.01 / h)$ and higher $t_{1 / 2}(13.063 \pm 0.36 \mathrm{~h})$, proposing an apparently sustained release of DDA from the polymer matrix which efficiently resulted in the sustained absorption of nano-DDA in vivo. These findings substantiated that the bioavailability of DDA was extended after the nanoencapsulation into PCL polymer matrix. The AUC of the nano-DDA (50 mg/kg) was $2631 \pm 6.98(\mathrm{~h} \mathrm{X} \mathrm{ng} / \mathrm{ml}$ ) and AUC of free DDA was $1293 \pm 7.51$ (h X ng/ml) which was about 2.03 times lesser than the nano-DDA, in spite of the lower dosage of nanoDDA administered as compared to the free DDA. This could be corroborated that the systemic exposure of nano-DDA was much better than free DDA [20].

Xie et al. reported that nano-encapsulated curcumin using poly (lactic-co-glycolic acid (PLGA) as a polymer exhibited 26.5 of AB\% whereas $\mathrm{AB} \%$ of native curcumin was $4.73 \%$ which could be substantiated that PLGA displayed a bioadhesive property which enhanced the contact with gastrointestinal tract thereby increasing its residence time and effective drug absorption [21]. A study was done by Chang et al., revealed that quercetin-loaded nano micelles exhibited a stability in gastric and intestinal fluids and did not exert any toxic effect on Caco-2 cells [22]. According to Wang et al., nanoparticles served as successful carriers for quercetin, resveratrol and curcumin to enhance their aqueous solubility, stability, bioavailability and target specificity. Nanoencapsulation protects the loaded drugs from degradation, hydrolysis and oxidation and provide a constant circulation time [20].

A recent study carried out in healthy Thai volunteers by Thailand researchers reported that the multiple doses of DDA resulted in the AUC, $\mathrm{C}_{\max }$ and $\mathrm{T}_{\max }$ of about $44.89 \mathrm{ng} / \mathrm{ml}, 0.95 \mathrm{~h}, 128.17$ (h X ng)/ml) respectively [8]. As reported by Yao et al. and Ye et al., DDA demonstrated an efflux transporter-mediated absorption mechanism $[23,24]$.

In general, the absorption of any bioactive compounds in the living system is not only dependent on its solubility but also permeability of the drug. Mostly in the gastrointestinal tract, active transport mechanism plays an important role in preventing absorption of molecules. Intracellular accumulation of natural or bioactive compound is prevented by the efflux transporters in the intestinal epithelia. P-glycoprotein (Pgp) family of transporters, expressed on brush border membrane, could enable the intestinal efflux of bioactive compounds dissolved in the bloodstream [8].

Hence, results from this pharmacokinetic study suggested that nanoencapsulated DDA demonstrated an enhanced bioavailability than its free form and proved to be a promising nanoparticle for slow and sustained drug delivery.

\section{CONCLUSION}

The in vivo oral bioavailability of DDA drives to a conclusion that DDA loaded PCL nanoparticles served as effective carriers of an antidiabetic drug which could be spectated with its slow and sustained drug delivery and augment absolute bioavailability. Hence, this study might pave a path to antidiabetic drug discovery with good therapeutic efficacy with minimal or no side effects. However, further studies using clinical trials may authorize the results obtained in this study.

\section{ACKNOWLEDGEMENT}

The authors sincerely thank Central animal house, SRM medical college hospital and research centre, Kattankulathur for providing animal house facilities.

\section{CONFLICTS OF INTERESTS}

The authors report no conflicts of interest

\section{REFERENCES}

1. Ooi JP, Kuroyanagi M, Sulaiman SF, Muhammad TST, Tan ML. Andrographolide and 14-deoxy-11, 12-didehydro-andrographolide inhibit cytochrome P450s in HepG2 hepatoma cells. Life Sci 2011;88:216-23.

2. Sattayasai J, Srisuwan S, Arkaravichien T, Aromdee C. Effects of andrographolide on sexual functions, vascular reactivity and serum testosterone level in rodents. Food Chem Toxicol 2010;48:1934-8.

3. Pholphana N, Rangkadilok N, Saehun J, Ritruechai S, Satayavivad J. Changes in the contents of four active diterpenoids at different growth stages in Andrographis paniculata (Burm. f.) Nees (Chuanxinlian). Chin Med 2013;8:1749-6.

4. Yoopan N, Thisoda P, Rangkadilok N, Sahasitiwat S, Pholphana $\mathrm{N}$, Ruchirawat S, et al. Cardiovascular effects of 14-deoxy11,12-didehydroandrographolide and Andrographis paniculata extracts. Planta Med 2007;73:503-11.

5. Wu TS, Chern HJ, Damu AG, Kuo PC, Su CR, Lee EJ, et al. Flavonoids and ent-labdane diterpenoids from Andrographis paniculata and their antiplatelet aggregatory and vasorelaxing effects. J Asian Nat Prod Res 2008;10:17-24.

6. Lee MJ, Rao YK, Chen K, Lee YC, Chung YS, Tzeng YM. Andrographolide and 14-deoxy-11,12-didehydroandrographolide from Andrographis paniculata attenuate high glucoseinduced fibrosis and apoptosis in murine renal mesangeal cell lines. J Ethnopharmacol 2010;132:497-505.

7. Panossian AG. Pharmacokinetic and oral bioavailability of andrographolide from Andrographis paniculata fixed combination Kan Jang in rats and human. Phytomedicine 2000;7:351-64.

8. Pholphana N, Panomvana D, Rangkadilok N, Suriyo T, Puranajoti P, Ungtrakul $\mathrm{T}$, et al. Andrographis paniculata: dissolution investigation and pharmacokinetic studies of four major active diterpenoids after multiple oral dose administration in healthy thai volunteers. J Ethnopharmacol 2016;194:513-21.

9. Zanchetta B, Chaud MV, Santana MHA. Self-emulsifying drug delivery systems (SEDDS) in pharmaceutical development. J Adv Chem Eng 2015;5:130.

10. Nagalakshmi K, Pooja YR, Praveenkumar I, Sujatha S. Fabrication, characterization, in vitro drug release and glucose uptake activity of 14-deoxy, 11, 12-didehydroandrographolide loaded polycaprolactone nanoparticles. Asian J Pharm Sci; 2003. Doi.10.1016/j.ajps.2017.02.003.

11. Szkudelski T. Streptozotocin-nicotinamide-induced diabetes in the rat. Characteristics of the experimental model. Exp Biol Med 2012;237:481-90.

12. Fröde TS, Medeiros YS. Animal models to test drugs with potential antidiabetic activity. J Ethnopharmacol 2008;115:173-83.

13. Wan S, Sun Y, Qi X, Tan F. Improved bioavailability of poorly water-soluble drug curcumin in cellulose acetate solid dispersion. AAPS PharmSciTech 2012;13:159-66.

14. Isadiatruti D, T Budiati, S Martodihardjo. Bioavailability study of a physical mixture of carbamazepine and amino acids. Asian J Pharm Clin Res 2015;8:592-5.

15. Shin SC, Choi JS, Li X. Enhanced bioavailability of tamoxifen after oral administration of tamoxifen with quercetin in rats. Int J Pharm 2006;313:144-9.

16. Chawla JS, Amiji MM. Biodegradable poly(epsiloncaprolactone) nanoparticles for tumor-targeted delivery of tamoxifen. Int J Pharm 2002;249:127-38.

17. Kamil A, Chen CYO, Blumberg JB. Nanotechnology and functional foods: effective delivery of bioactive ingredients. John Wiley and Sons, Ltd, Chichester, UK. 2015:158-74.

18. Sun H, Mei L, Song C, Cui X, Wang P. The in vivo degradation, absorption and excretion of the PCL-based implant. Biomaterials 2006;27:1735-40.

19. Dhana Lekshmi UM, Poovi G, Neelakanta Reddy P. In vitro observation of repaglinide engineered polymeric nanoparticles. Dig J Nanomater Biostructures 2012;7:1-18.

20. Gao Y, Li Z, Sun M, Li H, Guo C, Cui J, et al. Preparation, characterization, pharmacokinetics, and tissue distribution of curcumin nanosuspension with TPGS as a stabilizer. Drug Dev Ind Pharm 2010;36:1225-34. 
21. Xie $\mathrm{X}$, Tao $\mathrm{Q}$ Zou $\mathrm{Y}$, Zhang $\mathrm{F}$, Guo $\mathrm{M}$, Wang $\mathrm{Y}$, et al. PLGA nanoparticles improve the oral bioavailability of curcumin in rats: characterizations and mechanisms, J Agric Food Chem 2011;59:9280-9.

22. Chang KL, Tan B, Chang KL, Lim BK, Chiu GN. Perorally active nano micellar formulation of quercetin in the treatment of lung cancer perorally active nano-micellar formulation of quercetin in the treatment of lung cancer. Int J Nanomed 2012;7:651-61.

23. Yao Y, Liao QF, Xie ZY, Zeng YE, Ma Y. Absorption mechanism of dihydro andrographolide in human Caco-2 cell monolayer model. Chinese Tradit Herb Drugs 2011;42:1154-57.
24. Ye L, T Wang, L Tang, W Liu, Z Yang, J Zhou, et al. Poor oral bioavailability of a promising anticancer agent andrographolide is due to extensive metabolism and efflux by P-glycoprotein. J PharmSci 2011;100:5007-17.

\section{How to cite this article}

- Nagalakshmi K, Sujatha S, Alwin D. Dda loaded PCL nanoparticles enhances the oral bioavailability of DDA in diabetes-induced experimental rats. Int J Pharm Pharm Sci 2017;9(4):198-202. 